\title{
Entscheiden zwischen Kopf und Bauch
}

\author{
In medizinischen Berufen werden täglich unzählige und gewichtige Entscheidun- \\ gen getroffen. Der Beitrag zeichnet aus einer historischen Perspektive den Wandel \\ der Beschreibungen und Bewertungen dieser Entscheide nach.
}

Marianne Jossen

Korrespondenz: Marianne Jossen Stapferhaus Lenzburg Schloss

CH-5600 Lenzburg Tel. 0628884825

jossen[at]stapferhaus.ch

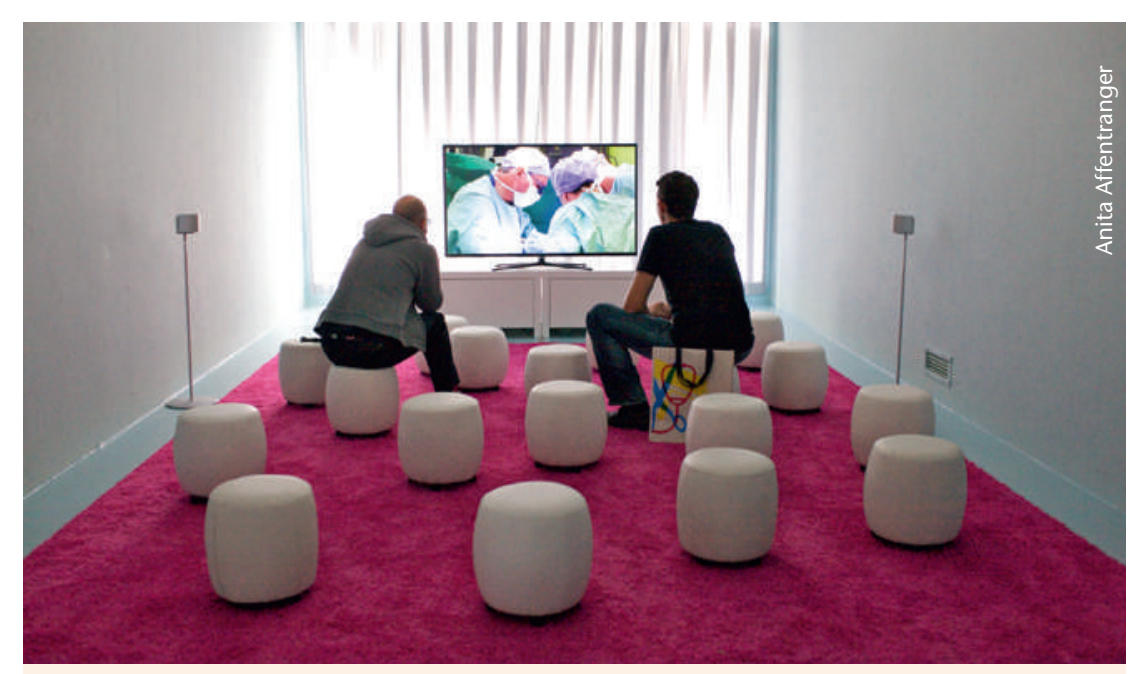

Eine Station in der Ausstellung des Stapferhauses.

«Grundsätzlich kann während einer Herzoperation immer etwas Unerwartetes auftreten, auch wenn vor dem Eingriff alle Informationen über den Patienten vorhanden sind. Denn man hat es in diesem Beruf mit einem Menschen zu tun, der ein Unikat ist. Und dieses Unikat verhält sich nicht immer nach unseren Wünschen», sagt der Herzchirurg Thierry Carrel in einem Videoporträt in der Ausstellung «Entscheiden» des Stapferhauses Lenzburg [1]. Medizinisches Entscheiden bewegt sich demnach zwischen einer mehr oder weniger umfassenden und präzisen Informationslage und einem konkreten Patienten mit seinem spezifischen Körper, seiner spezifischen Situation und der Zeit, die noch bleibt.

Diese «besonderen Merkmale ärztlichen Denkens» hat Ludwik Fleck bereits 1926 in einem Vortrag festgestellt. Der polnische Arzt und Immunologe, der im Lemberger Ghetto einen Impfstoff gegen Fleckfieber entwickelte, interessierte sich für Erkenntnis- und Entscheidungswege der Medizin. Auch er betonte dabei die Wichtigkeit von Information und vor allem der Statistik, merkte aber zugleich an, dass eine gewisse «spezifische Intuition» [2] hinzukommen muss, wenn es um das Zentrum medizinischen Interesses geht, nämlich um den konkreten Fall. Dabei spielt Fleck nicht direkt auf eine irgendwie geartete «Empathie» für den Patienten an. Die «Intuition» ist vielmehr eine Syntheseleistung, die Erfahrung, Geschick und Gefühl mit Informationen

Eine Station in der Ausstellung des Stapferhauses. zusammenbringt und so erst medizinisches Entscheiden möglich macht.

\section{Der Kampf gegen die Intuition}

Nach dem Zweiten Weltkrieg geraten Flecks Schriften in Vergessenheit. Es gewinnen andere Vorstellungen von Erkenntnis und Entscheidungsfindung in der Medizin und darüber hinaus an Gewicht: Die Nachkriegszeit ist die Zeit der Kybernetik. Nachdem das organizistische Menschenbild als den Holocaust mitverursachend identifiziert und verworfen wurde, fand die Kybernetik breiten Anklang in verschiedensten Bereichen, weil sie «eine gemeinsame Sprache zur Verfügung stellte und Verständigungsprozesse in Gang brachte» [2]. Doch was ist Kybernetik? Sie begreift Körper und Psychen, Staaten und Organisationen als Systeme - gesteuert von ihren Funktionen. Das Gesundheitswesen, die Praxis und die Klinik erfüllen die Funktion der Bereitstellung geheilter und arbeitsfähiger Bürger. An solchen Beschreibungen der Medizin als gesteuertes System entzündet sich die Idee, in diese Steuerung auch eingreifen zu können - im Sinne einer «Best practice», wo das Handeln unmittelbar und ohne Bruch aus dem Erkennen hervorgeht.

So entstehen in den 1950er Jahren in den USA Ansätze des Qualitätsmanagements für die Medizin. Sie machen aus dem Raum zwischen Gefühl und Kalkül eine deutliche Unterscheidung und schlagen sich auf die Seite der Zahlen, der wissenschaftlich geprüften Erkenntnis und des formalen Vorgehens. Überhaupt sagt man in dieser Zeit der Intuition, den alten Hierarchien und gewachsenen Autoritäten in Gesundheitsorganisationen und darüber hinaus den Kampf an. Sicher nicht zu unrecht, aber wohl auch, weil man sich zu dieser Zeit kaum vorstellen kann, wie ein möglicher Beitrag der Intuition zu medizinischer Qualität überhaupt gemessen werden könnte. Und so wurden und werden - mit gespaltenen Reaktionen bei den Professionellen - medizinische Entscheidungen zunehmend dokumentiert und systematisiert und damit hinterfragbar und begründungsbedürftig gemacht.

Am besten rechtfertigen wiederum lässt sich eine medizinische Entscheidung mit möglichst soliden wissenschaftlichen Fakten. Sie sollen dem medizinischen Handeln zugrunde liegen und so wesentlich zur Qualität im medizinischen Bereich beitragen. 
Die Diskreditierung von Intuition, Improvisation und Erfahrungswissen ist nicht denkbar ohne den rasanten Zuwachs medizinischer Forschung, die immer neue Zahlen, Statistiken und Risikoberechnungen produziert.

Sicher sind die Erkenntnisse der evidenzbasierten Medizin unverzichtbar in der Entscheidungsfindung. Gleichzeitig sind aber auch Intution und Erfahrung wichtig. Darauf zu verzichten oder diese zu verleugnen birgt eigene Risikien. Diese schildert der Psychologe Gerd Gigerenzer, Professor am Max Planck Institut für Bildungsforschung: Intuitive Entscheidungen werden noch immer getroffen, oftmals werden sie aber verschwiegen und im Nachhinein als wissenschaftlich gerechtfertigte Entscheidungen dargestellt. Gigerenzer fand zudem heraus, dass medizinisches Personal unter Rechtfertigungsdruck dazu tendiert, sich entgegen der eigenen Erfahrung und Intuition zu entscheiden - nicht immer zum Besten des Patienten [4]. Qualitätsmanagement und evidenzbasierte Medizin scheinen sich in gewissen Fällen selber zu sabotieren.

\section{Entscheidungen wurden zunehmend dokumentiert, systematisiert und damit hinterfragbar und begründungsbedürftig gemacht.}

\section{Literatur}

1 T. Carrel im Interview mit dem Stapferhaus Lenzburg.

2 Fleck L. Über einige besondere Merkmale ärztlichen Denkens. In: Schäfer L, Schnelle T (Hrsg.) Ludwik Fleck. Erfahrung und Tatsache. Frankfurt a. M.: Suhrkamp; 1983. S. 39.

3 Hagner M, Hörl G. Überlegungen zur kybernetischen Transformation des Humanen. Frankfurt a. M.: Suhrkamp; 2008. S. 13.

4 Gigerenzer G. Bauchentscheidungen, die Intelligenz des Unbewussten und die Macht der Intuition. 4. Aufl. München: Goldmann; 2008. Insb. S. 169ff
Wie der Herzchirurg Carrel zur Frage nach dem Qualitätsmanagement in der Ausstellung «Entscheiden» zu Protokoll gibt: «Das sind sinnvolle Konzepte, aber man kann sie nicht immer eins zu eins auf die Medizin übertragen. [1]

\section{Die Wiederentdeckung des Zwischenraums}

Carrels Aussage sucht nach einem Raum zwischen Verwaltung, Statistik und Einzelfall. Und genau dafür beginnen sich die Wissens- und Entscheidungstheorien in den 80er Jahren wieder zu interessieren. Fleck wird in dieser Zeit wiederentdeckt, und unzählige Studien aus der Philosophie, Psychologie, Soziologie, Ethnographie und auch aus dem Bereich des Qualitätsmanagements erkunden erneut die vielfältigen Schattierungen des Wissens und Entscheidens zwischen Emotion, Intuition und Information.

Gigerenzer etwa bezeichnet den Raum zwischen purer Intuition und reiner Statistik als «Heuristik». Das ist eine Entscheidungsstrategie, die in unübersichtlichen Situationen nicht die totale Berechenbarkeit sucht und doch wissenschaftlich unterfüttert ist. Es gilt, Zahl und Bauchgefühl zusammenzubringen.

Natürlich, so Gigerenzer, könne man bei einer Entscheidung darauf pochen, genauestens alle Risiken und Faktoren abzuwägen - und kreiert dann beispielsweise eine sogenannte «Entscheidungshilfe».
Viele von diesen angeblichen Hilfen werden aber im Alltag nicht benutzt, wenn es darum geht, rasch eine Entscheidung in einem spezifischen Fall zu treffen.

Gigerenzer erzählt in seinem Buch [4] die Geschichte einer Klinik in Michigan, die eine Entscheidungshilfe (das Heart Disease Predictive Instrument) einführte, um zu bestimmen, ob ein Patient mit Herzinfarktsymptomen in die Intensivstation oder in ein reguläres Bett gelegt werden sollte. Haargenau berechneten die Ärzte dabei mit einem Taschenrechner ein Dutzend Wahrscheinlichkeiten. Am Schluss stand eine Zahl, die eine Entscheidung bedeutete. Das Instrument wurde kaum akzeptiert, es war zu kompliziert. Erstaunlich war, dass die Ärzte - nachdem man ihnen die Entscheidungshilfe wieder weggenommen hatte - besser entschieden als vor der Einführung. Gigerenzers Erklärung: Die Ärzte arbeiteten wieder intuitiv, diesmal aber auf Grundlage einiger wichtiger und wissenschaftlich begründeter Faktoren, die ihnen aus der Arbeit mit der Entscheidungshilfe in Fleisch und Blut übergegangen waren.

Gigerenzer plädiert für eine «Intuitionswissenschaft», die solche Erkenntnisse ernst nimmt und mit ihrer Hilfe Intuition trainiert. Im Fall der genannten Klinik wurde schliesslich ein «effizienter Entscheidungsbaum» eingeführt: Nur die Kriterien, an die sich die Ärzte intuitiv hielten, wurden dort ihrer Wichtigkeit nach gegliedert. Nach jedem Kriterium kann es sein, dass die Entscheidung für die Intensivstation bereits fällt und der Rest des Entscheidungsbaums nicht abgearbeitet werden muss. So ein Entscheidungsbaum macht also Intuition - dieses Gespinst - auf gewisse Weise fassbar und damit auch vermittelbar.

Ob Gigerenzers Ansatz medizinisches Entscheiden verbessern kann - darüber müssen letztlich die Praktiker urteilen. Die kurze und aspekthafte Geschichte von Fleck bis heute mag aber zeigen, dass die alte Unterscheidung zwischen Kopf und Bauch selbst eine Heuristik sein könnte, die es uns erlaubt, unser Handeln und Entscheiden zu reflektieren, zu rechtfertigen und zu bewerten. Und dass es immer wieder Sinn macht, Heuristiken auch zu hinterfragen.

Zumindest wenn es ums Entscheiden in der Medizin geht, so scheint es, dass man versuchen könnte, die Zahl und die Intuition nicht gegeneinander sondern miteinander einzusetzen, um zu guten Entscheidungen zu gelangen.

Die Ausstellung «Entscheiden» des Stapferhauses Lenzburg führt die Besucher(innen) noch bis zum 25. April 2014 in den Supermarkt der Möglichkeiten: eine Welt, in der vieles möglich scheint, aber wenig sicher ist und in der wir täglich unzählige Kopf- und Bauchentscheidungen treffen. Auch Gerd Gigerenzer und Thierry Carrel kommen zu Wort. www.stapferhaus.ch 